\title{
Les modifications récentes du droit de la négociation collective en Grèce
}

\section{Costas Papadimitriou}

\section{(2) OpenEdition}

1 Journals

\section{Édition électronique}

URL : https://journals.openedition.org/rdctss/1690

DOI : $10.4000 /$ rdctss. 1690

ISSN : 2262-9815

Éditeur

Centre de droit comparé du travail et de la sécurité sociale

\section{Édition imprimée}

Date de publication : 1 avril 2019

Pagination : 218-221

ISSN : 2117-4350

\section{Référence électronique}

Costas Papadimitriou, "Les modifications récentes du droit de la négociation collective en Grèce »,

Revue de droit comparé du travail et de la sécurité sociale [En ligne], 1 | 2019, mis en ligne le 01 novembre 2021, consulté le 13 novembre 2021. URL : http://journals.openedition.org/rdctss/1690 ; DOI : https:// doi.org/10.4000/rdctss. 1690

\section{(c) (i) $\odot$}

Revue de droit comparé du travail et de la sécurité sociale est mise à disposition selon les termes de la Licence Creative Commons Attribution - Pas d'Utilisation Commerciale - Pas de Modification 4.0 International. 


\section{COSTAS PAPADIMITRIOU}

Université Nationale et Kapodistriague d'Athènes

\section{LES MODIFICATIONS RÉCENTES DU DROIT DE LA NÉGOCIATION COLLECTIVE EN GRÈCE}

Les modifications apportées, pendant la période de la crise économique, au droit collectif du travail grec ont été si importantes qu'il semble que sa physionomie ait été changée. Les points d'équilibre sur lesquels l'édifice des relations industrielles reposait ces dernières années, voire ces dernières décennies, ont été déplacés ${ }^{1}$. Parmi ces changements ceux liés à la hiérarchie des conventions collectives et au cadre de leur application gardent une importance particulière. Or, c'est au mois d'août 2018 que ces changements ont été renversés et certains principes traditionnels rétablis.

\section{I - LA HIÉRARCHIE DES CONVENTIONS COLLECTIVES}

Le système grec de négociation collective combine tous les niveaux de conventions: les négociations se déroulent aux niveaux, national, de la branche, de la profession et de l'entreprise. II existe ainsi différentes catégories de conventions collectives correspondant aux niveaux respectifs de négociation, telles que la convention nationale interprofessionnelle, qui englobe l'ensemble de l'économie, les conventions de branche, les conventions de métier et les conventions d'entreprise.

L'articulation de niveaux différents était, jusqu'en 2010, assurée par l'application du principe de faveur. Or, le cadre a été complètement modifié après la crise économique.

Selon le régime établi par la loi 1876/1990², en vigueur jusqu'en 2010, en cas de concours de conventions collectives de domaine d'application différent, c'était surtout le principe de faveur qui s'appliquait ${ }^{3}$. Selon les règles générales relatives à la solution de conflit entre plusieurs conventions collectives, on les comparait par unité (unité de salaire unité des clauses autres que le salaire) et on appliquait l'unité des clauses la plus favorable de la convention collective ou de la sentence arbitrale. Tout à fait exceptionnellement, s'il s'agissait d'une convention collective de branche ou d'entreprise, c'était ces clauses, mêmes si elles étaient moins favorables, qui s'appliquaient, quand elle concourait avec une convention collective de métier (art. 10 § 2 loi 1876/90). II s'agissait là d'une tentative du législateur de limiter le rôle du syndicalisme de métier, lié au corporatisme, au profit de celui

1 C. Papadimitriou, "Le droit grec face à la crise: un passage dangereux vers une nouvelle physionomie juridique », Revue de droit comparé du travail et de la sécurité sociale 2012/2 p. 6 et s.

2 Journal Officiel A/27/8-3-1990.

3 Sur le principe de faveur, voir G. Leventis, Droit du travail collectif, (en grec), 2006, DEN, Athènes, p. 488 et s. 
de l'entreprise ou de la branche. Une partie de la doctrine soutenait même que ce principe de faveur, bien ancré dans le système juridique grec, avait un fondement constitutionnel de sorte que le législateur ne puisse le supprimer ${ }^{4}$.

Ce régime de hiérarchie des conventions collectives avait pourtant subi, au cours des dernières années de la crise économique, une succession de modifications allant toutes dans le sens de l'abrogation du principe de faveur en cas de concours de conventions collectives, principe, on l'a déjà indiqué, ancré de longue date dans le droit du travail grec.

Deux lois, la loi $3845 / 2010$ et la loi 4024/2011, ont instauré la primauté de la convention collective d'entreprise. Ainsi, la priorité a été accordée à la convention collective d'entreprise, même si celle-ci contenait des clauses moins avantageuses que celles prévues par la convention de branche. La confirmation de l'abrogation du principe de faveur, de longue tradition en Grèce, a constitué une réalité, et la dérogation autorisée pouvait dès lors concerner toutes les matières ${ }^{5}$. II faut aussi ajouter que la légalité de la dérogation ne supposait pas que cela soit permis par la convention collective de rang supérieur ou d'invoquer un motif quelconque.

C'est ainsi, qu'il en a résulté une diminution de l'intérêt pour la conclusion de conventions collectives de branche, les organisations syndicales ayant été particulièrement conscientes de la possibilité de dérogation, à tout moment, par le biais de la négociation d'une convention d'entreprise.

Or, d'après le dernier accord de 2017 du gouvernement grec avec ses créanciers, celui-là a réservé son droit de réintroduire le principe de faveur au terme de la période de validité du Memorandum ${ }^{6}$. C'est alors à partir du mois d'août 2018 que cette modification a été introduite en droit grec.

De cette façon le droit collectif grec revient à ses origines, et plus particulièrement au principe de faveur en cas de concours entre convention collective de branche et convention collective d'entreprise. Ce changement de la structure de la négociation collective en Grèce étant encore en cours d'évolution, il faudra en observer les effets concrets dans les mois suivants.

\section{II - LE CADRE MATÉRIEL D'APPLICATION DES CONVENTIONS COLLECTIVES}

L'application matérielle de chaque convention collective dépend, selon le droit grec, de son niveau. Soit elle s'applique à tous les salariés intéressés, soit elle s'applique aux adhérents des organisations signataires. Dans le dernier cas le ministre disposait, jusqu'à 2012, du pouvoir d'étendre l'application de la convention.

4 A. Kazakos, Droit du travail collectif(en grec), 2013, Sakkoulas, Théssalonique, p. 54.

5 Voir $365^{\text {ème }}$ rapport du Comité de la liberté syndicale, $316^{\text {ème }}$ réunion, OIT: «la mise en place de procédures favorisant systématiquement la négociation décentralisée de dispositions dérogatoires dans un sens moins favorable que les dispositions de niveau supérieur peut conduire à déstabiliser globalement les mécanismes de négociation collective ».

6 Art. 16 de la Loi 4472/2017. 


\section{A - LE PRINCIPE}

L'application matérielle de chaque convention collective dépend, selon le droit grec, de son niveau. La convention nationale interprofessionnelle régit, en matière de salaire, uniquement les salaires des salariés des entreprises membres des organisations syndicales patronales signataires ${ }^{7}$. Pour toutes les autres matières, cette convention est susceptible d'être appliquée à l'ensemble des travailleurs du pays. Elle fixe les conditions minimales de travail pour tous les travailleurs indépendamment de leur affiliation syndicale. Les conventions collectives de branche et les conventions collectives de métier régissent normalement les relations de travail des adhérents aux organisations syndicales signataires. Enfin, la convention d'entreprise est appliquée à l'ensemble de son personnel, indépendamment de son affiliation syndicale. C'est ainsi que l'unité de réglementation des conditions de travail prévaut à ce niveau.

\section{B - L'APPLICATION ÉTENDUE DE CONVENTIONS COLLECTIVES DE BRANCHE ET DE MÉTIER}

Les conventions collectives de branche et de métier régissent normalement, on l'a déjà indiqué, les relations de travail des adhérents aux organisations syndicales signataires. Un cinquième environ des travailleurs grecs adhère à un syndicat. Le taux de syndicalisation est plus élevé dans le secteur public que dans le secteur privé où l'influence des syndicats est assez faible, notamment dans les petites et moyennes entreprises.

Toutefois, le Ministre du travail disposait du pouvoir d'accorder aux conventions collectives de branche et de métier une application étendue, c'est-à-dire d'imposer lorsque les conditions prévues à cet effet étaient remplies - leur application non seulement aux adhérents des organisations syndicales signataires, mais également aux salariés nonsyndiqués et aux entreprises non-membres des organisations patronales signataires. Cette faculté d'extension accordée au Ministre du travail a été, pendant la période de la crise économique récente, purement et simplement abrogée par la loi 4046/2012. Étant donné que le taux de syndicalisation était alors au niveau le plus bas de l'histoire sociale grecque, l'application des conventions collectives de branche a été, de ce fait, indubitablement limitée, diminuant d'autant leur importance. Or, d'après le dernier accord de 2017 du gouvernement grec avec ses créanciers, celui-là a également réservé son droit de réintroduire le pouvoir du ministre d'étendre l'application des conventions collectives aux non-adhérents au terme de la période de validité du Memorandum, c'est-à-dire à partir du mois d'août $2018^{8}$.

Ainsi, le Ministre du travail a, pour la première fois depuis 2012, suivi la voie d'extension des conventions collectives et quatre conventions ont été étendues en septembre 2018. Il faut pourtant signaler que cette fois le gouvernement doit suivre scrupuleusement les conditions prévues par la loi, c'est-à-dire vérifier que la convention, pour qu'elle puisse être étendue, régissent déjà les conditions de travail d'au moins $50 \%$ des salariés de la branche ou de la profession.

7 Les salaires minima des salariés dont les employeurs ne sont pas adhérents aux organisations syndicales patronales signataires de la convention nationale interprofessionnelle, sont fixés au niveau national par la loi.

8 Art. 16 de la Loi 4472/2017. 


\section{GRÈCE}

\section{Conclusion}

Les interventions liées à la législation de crise ont bouleversé non seulement la structure de la négociation collective, mais aussi l'ensemble des relations collectives grecques.

Or, une grande partie de ces interventions ont été, par la suite, modifiées. La reconnaissance de nouveau du principe de faveur, la réintroduction du pouvoir du ministre d'étendre l'application des conventions collectives de branche et de métier aux employeurs et aux salariés non affiliés aux organisations signataires et, enfin, le rétablissement en 2014 de l'arbitrage «obligatoire » et les modifications consécutives du régime de règlement des différends collectifs ont conduit à un changement considérable du cadre de la négociation collective et de ses effets. Des mesures introduites pendant la crise au régime grec des conventions collectives, il n'y a actuellement que la réduction de l'extension de la validité des clauses des conventions collectives au-delà de leur période d'application qui survive. 